\title{
Carbon Nanomaterials for Environmental Monitoring Sensors
}

\author{
Eduard Llobet \\ MINOS-EMAS, Universitat Rovira i Virgili, Avda. Paisos Catalans, 26, 43007 Tarragona, Spain \\ eduard.llobet@urv.cat
}

\begin{abstract}
:
Carbon nanomaterials are very promising for the design of new sensors able to detect airborne pollutants and the last years have seen a steadily growth of the research efforts and the publications on the use of such materials as sensitive films. In my talk I will review the main results obtained so far, identify some shortcomings and give an outlook.
\end{abstract}

Key words: carbon nanotubes, graphene, functionalization, nanoparticles, gas sensors, air pollutants.

\section{Introduction}

Pollution of the environment has been associated to industrialization. Contaminants can enter the ecosystems by many different pathways such as fugitive emissions and discharges from industry, municipal waste, runoff from agro-forestry applications or accidents. [1]. Air and water pollution are of high concern since airborne or water borne pollutants can be dispersed over enormous distances. According to the US Environmental Protection Agency, some of the most important air and water contaminants include particulate matter (PM), nitrogen oxides (NOx), ground level ozone, sulfur oxides (SOx), volatile organic compounds (VOCs) particularly benzene, toluene, ethylbenzene and xylenes (BTEX), carbon monoxide, chlorofluorocarbons (CFCs) arsenic, lead, mercury, microbial pathogens or endocrine disruptors, just to mention a few [2].

Traditionally, instrumental methods are the tools used to detect compounds of interest and to track their occurrence in the environment [1]. This involves the periodical sampling and its chemical analysis by gas chromatographymass spectrometry (GC-MS) or liquid chromatography-MS [3]. As a result, a small number of hazardous chemicals or pathogens are actually monitored and a continuous and widespread monitoring is impossible, since the labor and transport associated are far too costly. This is why sustained research efforts have been directed towards the development of gas sensors and biosensors, as essential tools for implementing the European Union Directives on environmental protection.
In the framework of this constant effort to develop miniaturized sensors with enhanced performance, the last years have seen a steadily growth of the use of nanomaterials as sensitive films. Among these, carbon nanomaterials such as carbon nanotubes, graphene or graphene oxide have been widely studied both in gas and bio sensing applications that are relevant to environmental monitoring. Therefore, the objective of this talk is to critically review the literature, present the main results, identify shortcomings and outline the prospects of carbon nanomaterial sensors for integrating a new generation of environmental assessment and monitoring systems. Given the fact that the COST action EuNetAir is centered on new sensors for the monitoring of air pollution, this review will be focused on carbon nanomaterial based gas sensors.

\section{Carbon nanomaterials}

In the last few years, different studies have shown the excellent potential of carbon nanotubes (CNTs) as sensitive material for detecting biological and chemical molecules $[4,5]$. Via a functionalization of CNT sidewalls, a better chemical bonding between a specific chemical species and the nanotube can be reached and the selectivity of the adsorption process can be enhanced [4,5]. Some properties of CNTs make them very attractive to produce small, wearable sensors for environmental monitoring. Their intrinsic strength makes them suited for miniaturized sensors and usable on flexible substrates. They respond even when operated at room temperature, which is optimal for ultra-low power, wearable, battery-operated devices. The 
adsorption of a small quantity of chemical species can result in a dramatic change of the CNT conductivity. Therefore, CNT are suited to detect species at low concentrations (e.g. low ppb level). A sensor can be built using a simple transducer (comb electrodes) to monitor the electrical resistance of a CNT-based film or, alternatively, CNTs can integrate the channel of a FET device [6].

A few properties found in graphene make it very promising as sensitive material. It is a twodimensional material and has its whole volume exposed to surface adsorbates. Graphene is highly conductive, exhibiting metallic conductivity and, hence, low Johnson noise even in the limit of no charge carriers, where a few extra electrons can cause notable relative changes in carrier concentration. Graphene has few crystal defects, which ensures a low level of excess $(1 / f)$ noise caused by their thermal switching. And, finally, graphene allows fourprobe measurements on a single crystal device with electrical contacts that are ohmic. All of these features contribute to make a unique combination that maximizes the signal-to-noise ratio to a level sufficient for detecting changes in a local concentration by less than one electron charge, $e$, at room temperature [7].

\section{Gas sensors employing carbon nanomaterials}

Once the interesting properties of the different carbon nanomaterials have been discussed, a critical review of carbon nanomaterial gas sensors will be given. In particular, device architectures, growth, functionalization and integration of nanomaterials and device performances (sensitivity, selectivity, baseline recovery and stability) will be compared. Special attention will be given to review methods for creating defects and grafting functional groups and metal nanoparticles to carbon nanomaterials in view of tuning their reactivity to target molecules $[6,8]$.

Good response of carbon nanomaterials to some environmental monitoring relevant chemical species such as CO, NOx or VOCs has been reported. As an illustrative example, Fig. 1 shows the detection of benzene traces in air employing an array of carbon nanotube sensors decorated with different metal nanoparticles. The limit of detection is below 50 $\mathrm{ppb}$ and the sensor array is operated at room temperature. The different metal nanoparticles show different reactivity for benzene. For example, when benzene adsorbs on a Rh nanoparticle, there is a significant amount of charge transferred between the Rh cluster and the carbon nanotube, in such a way that the conductivity of the carbon nanotube is affected. This change in conductivity can be easily measured (e.g. using interdigited electrodes). It is the combination of benzene-sensitive and benzene-insensitive materials that gives rise to selectivity $[9,10]$.
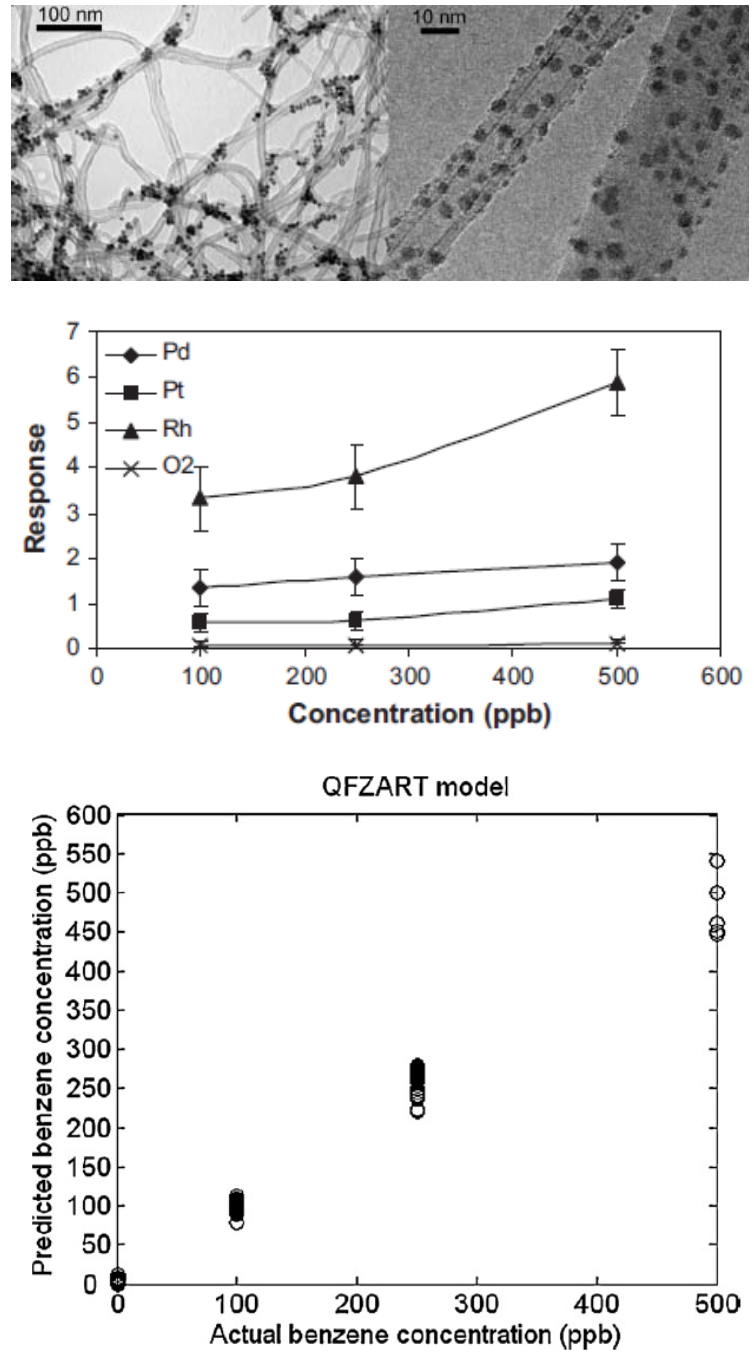

Fig. 1. TEM images of multiwalled carbon nanotubes decorated with $\mathrm{Rh}$ (left) and Pf (right) nanoparticles. Response to benzene vapors of resistive sensors employing either CNTs decorated with different metal nanoparticles or bare oxygen plasma treated CNTs. Quantitative model for predicting benzene concentration in the presence of interfering species and humidity. Adapted from $[6,10]$.

Hybrid nanomaterials based on multiwall carbon nanotubes decorated with tin oxide nanoclusters (about $5 \mathrm{~nm}$ in diameter) synthesized by the precipitation of a $\mathrm{SnO}_{2}$ colloidal suspension in the presence of carbon nanotubes have been reported [11]. Hybrid nanomaterials were prepared with different carbon nanotube to tin oxide ratios. When an appropriate ratio between the amount of plasma treated nanotubes and tin oxide precursors is employed, hybrid sensors show superior 
performance in the detection of nitrogen dioxide in the ppb range and carbon monoxide in the low ppm range. Furthermore, these optimized sensors show a significantly reduced moisture cross-sensitivity.

Nitrogen or boron-substituted carbon nanotubes have been reported as well. N-doped CNTs are efficient at detecting hazardous gaseous species because of the presence of pyridinetype sites on their surface. Villalpando-Paez and co-workers [12] found that nitrogen doped MWCNTs sensors exhibited response and recovery times of the order of a few seconds to $\mathrm{NH}_{3}$.

K.S. Novoselov and co-workers [7] studied graphene devices that were prepared by micromechanical cleavage of graphite at the surface of oxidized Si wafers. This allowed them to obtain graphene monocrystals of typically $10 \mu \mathrm{m}$ in size. By using electron-beam lithography, they made electrical (Au/Ti) contacts to graphene. The conductance of the devices to $\mathrm{NO}_{2}, \mathrm{NH}_{3}, \mathrm{H}_{2} \mathrm{O}$ and $\mathrm{CO}$ in concentrations, $\mathrm{C}$, of 1 part per million (p.p.m.) in nitrogen or helium were investigated. Large detectable changes occurred within $1 \mathrm{~min}$ after letting the chemicals in. The baseline was regained by heating at $150^{\circ} \mathrm{C}$, which helped removing species adsorbed on graphene. NO2, $\mathrm{H}_{2} \mathrm{O}$ and iodine acted as acceptors, whereas $\mathrm{NH}_{3}, \mathrm{CO}$ and ethanol were donors. Hall measurements demonstrated the possibility of monitoring single-molecule adsorption events.

Carbon nanomaterials show very interesting properties for designing room-temperature operated gas sensors able to detect traces of air pollutants such as nitrogen oxides, carbon monoxide or volatile aromatic compounds. However, more efforts are needed in order to better understand their sensing mechanisms, to reliably control their surface properties and to obtain their facile integration in gas-sensitive devices.

\section{Acknowledgements}

This work has been funded in part by the Catalan Agency for Research under grant no. 2011 CTP 00015 and by the European Science Foundation under COST TD1105 'EuNetAir'.

\section{References}

[1] T.Kosjek, E. Heath, M. Petrovic, D. Barcelo, Mass spectrometry for identifying pharmaceutical biotransformation products in the environment, Tends Anal. Chem 26, 1076-1085- (2007); doi:10.1016/j.trac.2007.10.005

[2] US Environmental Protection Agency (http://www.epa.gov)
[3] C. Blasco, Y. Pico, Prospects for combining chemical and biological methods for integrated environmental assessment, Trends Anal. Chem. 28, 745-757 (2009); doi: 10.1016/j.trac.2009.04.010

[4] P. Bondavalli, P. Legagneux, D. Pribat, Carbon nanotubes based transistors as gas sensors: state of the art and critical review Sens Actuators B: Chem, 140, 304-318 (2009); doi:10.1016/j.snb.2009.04.025

[5] C. Li, ET. Thostenson, TW. Chou, Sensors and actuators based on carbon nanotubes and their composites: a review. Compos. Sci Technol, 68 1227-1249 (2008); doi:

10.1016/j.compscitech.2008.01.006

[6] R. Leghrib, A. Felten, F. Demoisson, F. Reniers, JJ. Pireaux, E. Llobet, Room-temperature, selective detection of benzeneat trace levels using plasma-treated metal-decorated multiwalled carbon nanotubes, Carbon, 48, 34773488 (2010); doi: 10.1016/j.carbon.2010.05.045

[7] F. Schedin, A. K. Geim, S. V. Morozov, E. W. Hill, P. Blake, M. I. KatsnelsonAnd K. S. Novoselov, Detection of individual gas molecules adsorbed on graphene, Nature Mater. 6, 652-655 (2007); doi: 10.1038/nmat1967.

[8] M. Penza, R. Rossi, M. Alvisi, M.A. Signore, G. Cassano, D. Dimaio, R. Pentassuglia, E. Piscopiello, F. Serra, M. Falconeri, Characterization of metal-modified and verticallyaligned carbon nanotube films for functionally enhanced gas sensor applications, Thin Solid Films, 517, 6211-6216 (2009); doi: 10.1016/j.tsf.2009.04.009

[9] Z. Zanolli, R. Leghrib, A. Felten, J.J. Pireaux, E. Llobet, J.C. Charlier, Gas-sensing with Audecorated carbon nanotubes, ACS Nano, 5, 4592-4599 (2011); doi: 10.1021/nn200294h

[10] R. Leghrib, E. Llobet, Quantitative trace analysis of benzene using an array of plasma-treatemetaldecorated carbon nanotubes and fuzzy adaptive resonant theory techniques, Anal. Chim. Acta, 708, 19-27 (2011); doi:10.1016/j.aca.2011.09.038

[11] R. Leghrib, R. Pavelko, A. Felten, A. Vasiliev., C. Cané. I Gràcia, JJ. Pireaux, E Llobet, Gas sensors based on multiwall carbon nanotubes decorated with tin oxide nanoclusters, Sensors and Actuators B 145, 411-416 (2010); doi:10.1016/j.snb.2009.12.044

[12] F. Villalpando-Paez, A.H. Romero, E. MunozSandoval, L.M. Martinez, H. Terrones, M. Terrones, Fabrication of vapor and gas sensors using films of aligned $\mathrm{CN}_{x}$ nanotubes, Chem. Phys. Lett. 386, 137-143 (2004); doi:10.1016/j.cplett.2004.01.052 